\section{LOS CUIDADOS PALIATIVOS EN EL FINAL DE LA VIDA. ASPECTOS CLÍNICOS Y ÉTICOS}

\author{
Victoria Espinar Cid \\ Hospital Doctor Moliner (Valencia) \\ ORCID: https://orcid.org/0000-0001-5738-4277 \\ espinar_vic@gva.es
}

\begin{abstract}
Cómo citar este artículo/Citation: Espinar Cid, V. (2019). Los cuidados paliativos en el final de la vida. Aspectos clínicos y éticos. Arbor, 195 (792): a504. https://doi.org/10.3989/
\end{abstract} arbor.2019.792n2005

Recibido: 30 septiembre 2015. Aceptado: 28 abril 2016.

RESUMEN: El alivio del sufrimiento en el periodo final de la vida, reconocido en los últimos años como un derecho universal del ser humano, es una de las misiones de la medicina. La reflexión ética en la toma de decisiones en este periodo final de la vida constituye uno de los fundamentos de la identidad de los cuidados paliativos. Para dar respuesta a las múltiples necesidades de pacientes y familias es imprescindible una actitud empática, solidaria y respetuosa. Esta filosofía humanista debe ir complementada con los avances científicos que la medicina ha experimentado en las últimas décadas.

PALABRAS CLAVE: Cuidados paliativos; final de vida; sufrimiento; eutanasia.

\section{PALLIATIVE CARE AT THE END OF LIFE. CLINICAL AND ETHICAL ASPECTS}

Copyright: (C) 2019 CSIC. Este es un artículo de acceso abierto distribuido bajo los términos de la licencia de uso y distribución Creative Commons Reconocimiento 4.0 Internacional (CC BY 4.0).

ABSTRACT: Alleviating suffering in the last days of life is being recognised in recent years as a universal human right and as one of the major purposes of medical practice. Ethical reflection on decision-making in this final stage of life is one of the cornerstones of the identity of palliative care. In order to meet the needs of patients and their relatives, an empathetic, caring and respectful attitude is vital. The scientific achievements that medical science has accomplished in the last decades must be complemented by this humanistic philosophy.

KEYWORDS: Palliative care; end of life; suffering; euthanasia. 
Los que sufren no son los cuerpos, son las personas

(Casell, 2004).

\section{INTRODUCCIÓN}

Nacer y morir es parte de la historia natural de la especie humana y de cada persona concreta. Cuidar, acompañar esos dos momentos de nuestra vida es igual de importante hoy, y así lo ha sido desde tiempos remotos. El siglo XX ha conocido el auge de las ciencias de la salud en investigación, tecnología diagnóstica y tratamientos. Todo ello ha permitido un mayor control de las enfermedades y, por ende, ha prolongando la esperanza de vida. Es saber común en medicina que más vale prevenir que curar y que si no podemos curar quedan todavía muchos cuidados que ofrecer a la persona enferma. No obstante, los cuidados de las personas en el final de su vida han estado relegados a un segundo plano. En muchos ámbitos, incluido el de las profesiones sanitarias, y en la sociedad en general, se les ha restado importancia. Pero en las últimas décadas del siglo XX dos mujeres, la enfermera Cicely Saunders (19182005) y la psiquiatra Elisabeth Kubler-Ross (1926-2004) lanzaron un mensaje contundente de trabajo y de actitud personal en los cuidados de las personas al final de la vida. Con su ejemplo, todos los que trabajamos en cuidados paliativos (CP) hemos ido avanzando, formándonos, investigando en todas las facetas de las personas enfermas para restaurar el lugar que deben ocupar estos cuidados en la sociedad en general y en las profesiones sanitarias. Así nacieron y se desarrollaron los cuidados paliativos, que han formado parte de nuestro trabajo día a día... Y seguimos luchando por mejorarlos y transmitir su filosofía, su buen hacer y su abordaje multidisciplinar a las nuevas generaciones.

El desarrollo y difusión de los CP es, como decimos, reciente, e incluso en los últimos años se ha apoyado decididamente en el uso de las redes sociales de Internet. No obstante, en realidad, la medicina paliativa parte de una concepción muy antigua de la medicina, que toma como su objeto de estudio a la persona enferma en su conjunto. La actitud paliativa no es antagónica de la actitud curativa, sino complementaria de esta. A medida que la enfermedad va prolongándose en el tiempo cambian los objetivos médicos y van cobrando más relieve el alivio de los síntomas y de las secuelas, así como el cuidado del enfermo para hacerle más llevadero el curso final de la enfermedad. La medicina paliativa parte, pues, de la premisa de que "siempre hay algo que hacer", ya que es posible aliviar el sufrimiento del paciente aun en la fase terminal.
Por diferentes motivos, los CP se han desarrollado sobre todo en la atención a pacientes oncológicos, pero el aumento de la frecuencia de patologías crónicas, que también pueden evolucionar hacia una situación de enfermedad avanzada o terminal, está generando necesidades múltiples y una demanda asistencial elevada a la que es necesario dar respuesta científica de calidad, abordando, de forma multidisciplinar, los aspectos físicos, emocionales, psicológicos, sociales, espirituales y éticos.

Al hablar de aspectos sociales, nos referimos a que la vida familiar se modifica de manera sustancial ante una enfermedad grave o avanzada, lo cual exige el desarrollo de mecanismos que permitan la adaptación de cada uno de sus miembros a la nueva situación. La enfermedad, los tratamientos, y sobre todo la posibilidad de una muerte cercana transformarán el ambiente familiar generando un clima de fuerte tensión emocional. Por ello, los CP han de incluir la intervención emocional al final de la vida, que tiene como objetivo disminuir la sensación de amenaza, potenciar los recursos y compensar o modificar el estado de ánimo cuando sea necesario.

En este terreno, la sociedad ha ido madurando en los últimos años a través de diversos debates éticos. Pero la facilidad y rapidez con la que la información se difunde en la actualidad hace que tengamos poco tiempo para la reflexión profunda sobre algunos temas que atañen a nuestra vida. Este es el caso de las decisiones en el final de la misma. ¿En que momento y circunstancia nos detenemos a pensar en la muerte? Formar un juicio reflexivo sobre el final de nuestra propia vida constituye, en realidad, una expresión de autonomía y de libertad, del deseo de cada persona de tener una buena muerte y de que los demás reconozcan y acepten esta autonomía.

A veces se considera que las peticiones de eutanasia o de suicidio asistido constituyen también una expresión de autonomía. Para aclarar este punto, hay que recordar que el concepto de eutanasia debe incluir exclusivamente la acción u omisión, directa e intencionada, encaminada a provocar la muerte de una persona que padece una enfermedad avanzada o terminal, a petición expresa y reiterada de esta. Por su parte, el suicidio asistido consiste en la ayuda intencionada a la realización de un suicidio, ante la demanda de un enfermo competente que no pueda realizarlo por sí mismo debido a limitaciones de carácter físico. Sabemos que estas situaciones pueden plantearse en algún momento de nuestra trayectoria personal o profesional. Es necesario, pues, tenerlo bien trabaja- 
do en todos los aspectos, desde la experiencia clínica, la evidencia científica, la reflexión ética y la normativa legal para dar una respuesta adecuada. La experiencia nos dice que la expresión del deseo de morir suele ser una petición de auxilio de un ser humano que sufre física, psíquica, emocional, social y espiritualmente. Precisamente una asistencia paliativa integral puede aliviar este "dolor total", dar respuesta adecuada a esa petición de auxilio y reconducir el deseo de morir hacia una muerte tranquila y serena. Lo que nunca debería de pasar es que un paciente solicite la eutanasia porque no se sabe o no se pueden utilizar los multiples recursos disponibles para aliviar su sufrimiento.

La implementación de un modelo que dé respuesta a los problemas de todos los enfermos en el final de sus días, la creación y organización de recursos asistenciales adecuados y la formación específica de los profesionales implicados son fundamentales para conseguir el objetivo principal de los CP, a saber, el de mejorar la calidad de vida de los enfermos al final de sus vidas por medio de una mejora en la calidad de la atención.

\section{ASPECTOS GENERALES DE LA ATENCIÓN AL FINAL DE LA VIDA}

\subsection{Las razones para el desarrollo de los $\mathrm{CP}$}

Existen poderosas razones demográficas, epidemiológicas y sociales que hacen necesario el desarrollo de los CP y que podemos resumir en:

- Aumento de la esperanza de vida en las últimas décadas. Esta situación conlleva un progresivo envejecimiento de la población, de forma que actualmente en España el número de personas mayores de 65 años supone el $16 \%$ de la población y se estima que alcanzará el $20 \%$ en los próximos veinte años. Este patrón demográfico condiciona el aumento de la prevalencia de patologías crónicas, degenerativas y tumorales que pueden evolucionar hacia una situación clínica de enfermedad terminal, incrementando de manera muy importante la demanda de atención sociosanitaria generada por los principales problemas de salud de este grupo poblacional. Distintos estudios estiman que la prevalencia de las enfermedades crónicas avanzadas oscila entre 350 y 1.400 pacientes por millón de habitantes. En referencia directa al cáncer, está suficientemente establecido que la edad avanzada es un factor vinculado a una mayor prevalencia y mortalidad de las enfermedades neoplásicas. En España el $65 \%$ de las personas que fallecen por cáncer son mayores de 70 años. Es sobradamente conocida la magnitud del cáncer como problema de salud. De hecho, según datos del Instituto Nacional de Estadística (INE), el cáncer es la segunda causa de muerte en España, por detrás de las enfermedades cardiovasculares.

- Atención inadecuada. El alivio del sufrimiento en un paciente con una enfermedad en fase terminal requiere una estructura de recursos adecuada, un enfoque asistencial integral con personal formado y competente, que tenga además gran sensibilidad para acercarse a los problemas de enfermos y familiares. El estudio SUPPORT puso de manifiesto, por una parte, las múltiples situaciones de obstinación terapéutica que sufren los enfermos y, por otra, el abandono terapéutico al que muchos pacientes se ven sometidos en relación con determinados problemas. Aún hoy, los recursos sociosanitarios que provee el actual sistema de salud son insuficientes e inadecuados para dar respuesta a los problemas que, tanto cuantitativa como cualitativamente, plantean los pacientes y sus familias en situación de terminalidad. La falta de formación y las dificultades de integración entre los diferentes recursos implicados en cuidados paliativos justifican la necesidad de seguir abogando por la implantación de unos cuidados paliativos de calidad (Connors et al., 1995).

- Sensibilización social. Existe un interés creciente en nuestra sociedad por aspectos relacionados con la autonomía de la persona y con las decisiones en el final de la vida. En el debate público y profesional, y desde múltiples puntos de vista, cada vez es mayor el reconocimiento del derecho a una muerte con dignidad y al alivio del sufrimiento de todo enfermo en situación terminal. Esto ha llevado a que los diferentes gobiernos incluyan en sus programas acciones específicas en materia de planificación y legislación, que contemplen aspectos clínicos y éticos en el cuidado del paciente al final de la vida (planes de humanización de la asistencia, testamento vital, documento de voluntades anticipadas, comités de bioética).

\subsection{La definición de los CP}

El Subcomité Europeo de Cuidados Paliativos de la Comunidad Económica Europea definió en mayo de 1991 los CP como la asistencia total, activa y continuada a los pacientes y a sus familias por parte de un equipo multiprofesional cuando la expectativa médica no es la curación. La meta fundamental es dar calidad de vida al paciente y 
a su familia sin intentar alargar ni acortar la supervivencia, debiendo cubrir las necesidades físicas, psicológicas, espirituales y sociales del paciente y de sus familiares; incluso, si es necesario, el apoyo debe incluir el proceso de duelo.

Esta definición es compartida por la Organización Mundial de la Salud (OMS), que además añade que se trata de una ciencia médica y humana que reafirma la vida y ve la muerte como un proceso normal. En referencia a los enfermos de cáncer, la OMS destaca que, sean cuales fueren las posibilidades de curación del paciente, el alivio del dolor y los cuidados paliativos se deben considerar como elementos integrales y esenciales de todo programa nacional de lucha contra el cáncer (véase Organización Mundial de la Salud. Control del cáncer: Cuidados paliativos. Guía de la OMS para desarrollar programas eficaces).

El marco de actuación de los CP, tal y como se describe en la Estrategia de Cuidados Paliativos del Sistema Nacional de Salud, es la atención al paciente con enfermedad avanzada, incurable, progresiva y sin posibilidades razonables de respuesta al tratamiento específico, que provoca problemas como la presencia de síntomas multifactoriales, intensos y cambiantes, con la existencia de una gran impacto emocional en enfermos, familiares y equipos de cuidado y con un pronóstico de vida limitado en el tiempo, generalmente inferior a los seis meses. El principal objetivo de dicha atención es la mejora de la calidad de vida y el confort, definidos estos en acuerdo con el propio enfermo y su familia, con respecto a su sistema de creencias, preferencias y valores.

\subsection{Principios de los CP}

Siguiendo las recomendaciones de la Sociedad Española de Cuidados Paliativos (SECPAL) las bases de la terapéutica en pacientes terminales serán:

- El enfermo y la familia son la unidad a tratar. La familia es el nucleo fundamental del apoyo al enfermo, adquiriendo una relevancia especial en la atención domiciliaria.

- La promoción de la autonomía y dignidad del paciente tienen que regir en las decisiones terapéuticas. Esto solo será posible si se comparten con el enfermo los objetivos que se pretende conseguir.

- Concepción terapéutica activa, incorporando una actitud rehabilitadora y activa en la realización de los cuidados, superando el "no hay nada más que hacer".

- Importancia del ambiente. Una atmosfera de respeto, confort, soporte y comunicación influye de manera decisiva en el control de síntomas.
- Atención integral que tenga en cuenta los aspectos físicos, emocionales, sociales y espirituales. Forzosamente se trata de una atención individualizada y continuada.

La calidad de vida y el bienestar de nuestros pacientes antes de su muerte pueden ser mejorados considerablemente mediante la aplicación de los conocimientos actuales de los $\mathrm{CP}$, cuyos instrumentos básicos son:

- Control de síntomas: saber reconocer, evaluar y tratar adecuadamentse los numerosos síntomas que aparecen y que inciden directamente sobre el bienestar de los pacientes.

- Apoyo emocional y comunicación con el paciente, familia y equipo terapéutico, estableciendo una relación franca y honesta.

- Cambios en la organización, que permitan el trabajo interdisciplinar y una adpatación flexible a los objetivos cambiantes de los enfermos.

- El equipo interdiciplinar, sin el cual sería difícil ofrecer unos cuidados de calidad.

Es importante disponer de conocimientos y habilidades en cada uno de estos apartados, que constituyen verdaderas disciplinas científicas (Centeno Cortés, Gómez Sancho, Nabal Vicuña y Pascual López, 2009).

\subsection{Un modelo asistencial para los CP}

El modelo asistencial que se propone para desarrollar una atención de calidad al paciente susceptible de recibir cuidados paliativos, se caracteriza por ser:

- Integral, continuo e individualizado: con una visión holística del paciente (biopsicosocial y espiritual), que facilite el soporte necesario durante todo el proceso de la enfermedad y que dé respuesta individualizada a los problemas, considerando los principios, valores y preferencias de los propios pacientes y de sus familias.

- Integrado en la red asistencial del sistema sanitario: se trata de optimizar al máximo los recursos ya existentes y de integrar dentro de la red asistencial recursos específicos que atiendan preferentemente a pacientes con enfermedad avanzada y terminal, sobre todo cuando los niveles de complejidad lo requieran. Estos recursos estarán compuestos por equipos interdisciplinares con formación específica, protocolos y documentos de trabajo específicos, así como actividades de investigación y docencia 
apropiadas. Dichos equipos deberán ser reconocidos e identificados como referentes por los usuarios y las organizaciones.

- Coordinado en los niveles y servicios de atención especializada (hospitales de agudos y hospitales de media y larga estancia) y de atención primaria (equipos y modelo tradicional y unidades de apoyo domiciliario), y entre estos dos niveles. Dadas las múltiples y cambiantes necesidades de los enfermos es fundamental coordinar los diferentes recursos en cada área de atención, creando un circuito asistencial flexible, de atención compartida y corresponsabilidad entre los diferentes niveles y especialidades implicadas.

- Abierto a la coordinación con los recursos sociales (servicios sociales, voluntariado y ONGs)

- $\quad$ Formado por equipos interdisciplinares y multiprofesionales: la atención al paciente con enfermedad en fase terminal no podrá ser prestada por una sola disciplina profesional, sino que requerirá la participación de diferentes profesionales, sanitarios y no sanitarios, y de un trabajo en equipo en el que, aun desarrollando cada uno tareas específicas de su especialidad, se vean en la necesidad de solapar sus áreas de intervención y de adquirir unos conocimientos y habilidades interdisciplinares que permitan un adecuado cuidado (Sociedad Española de Cuidados Paliativos SECPAL, 2016).

\subsection{Estrategia terapéutica del control de síntomas}

Las palabras tantas veces repetidas "no hay nada que hacer", indican un desconocimiento importante de la enfermedad en situación terminal. Las consecuencias para el enfermo son tremendamente negativas, ya que implican o bien el abandono sanitario o bien la obstinación terapéutica. Este fatalismo aumenta el sufrimiento del paciente, quien tiene la sensación de que nada puede aliviarlo.

El control de los síntomas físicos es uno de los pilares más importantes de los CP. Estos síntomas son múltiples, cambiantes e intensos y tienden a aislar al paciente del mundo exterior. Pero no se debe olvidar que existen factores que los aumentan o disminuyen. Así, es bien conocido que el umbral del dolor disminuye con el insomnio, ansiedad, miedo o abandono social.

Frecuentemente se equipara sufrimiento con dolor físico, cuando el sufrimiento no es un síntoma sino una experiencia emocional. No se puede aliviar el sufrimiento con medicamentos. La búsqueda del control del sufrimiento como si se tratase de un síntoma es un obstáculo para su alivio. La morfina no puede aliviar la vulnerabilidad, el abandono y la marginación del paciente con enfermedad en fase terminal. Es más, a veces la liberación del dolor o la disnea puede acompañarse de mayor sufrimiento, ya que facilita la capacidad de reflexión del paciente sobre su situación. Es fundamental, por tanto, aprender a utilizar positivamente también el gran poder terapéutico que nuestra presencia tiene para los pacientes. En las situaciones de difícil control, será siempre algo que nos ayudará a aliviar y dar seguridad al enfermo.

Los pacientes con enfermedad en fase terminal, presentan durante el último mes de vida múltiples síntomas, con una media de 8,7 problemas por paciente. En una revisión realizada en la comunidad, los síntomas más prevalentes fueron: astenia, anorexia, somnolencia, dolor, estreñimiento y sequedad de boca, que estaban presentes en más del $50 \%$ de los enfermos. Desde un punto de vista práctico, en primer lugar, hay que conseguir el mejor control posible de los síntomas físicos; solo entonces podremos plantearnos disminuir la angustia, mejorar la depresión y la desesperanza y ayudar al enfermo a encontrar algún sentido a su vida pasada, a su situación actual y quizá a buscar una respuesta personal a su sufrimiento.

En este sentido, las recomendaciones de la Sociedad Española de Cuidados Paliativos para el alivio de síntomas físicos son las siguientes:

- Hacer un diagnostico fisiopatológico del síntoma. Los tratamientos deben basarse en el mecanismo de producción de los síntomas. En el caso del cáncer hay que evitar atribuir todos los síntomas al mismo, e investigar si se deben a los tratamientos administrados, a la debilidad o a procesos concomitantes. Se debe evaluar también su intensidad, el impacto físico y emocional que ocasionan y los factores que los aumentan o disminuyen. Suele ser inapropiado utilizar métodos diagnósticos muy agresivos para determinar los mecanismos de los síntomas. Debe combinarse adecuadamente la técnica con la sensibilidad para determinar las exploraciones indicadas.

- Explicar las causas de los síntomas al paciente y a su familia. Por supuesto, esto debe hacerse utilizando un lenguaje comprensible para ellos. Se les explicará la estrategia que se va a utilizar para el alivio de los síntomas. La explicación de estas medidas hace que aumente la confianza del enfermo y ejerce ya una 
acción terapéutica. En el esfuerzo terapéutico hay que valorar el interés, la voluntad y la energía del paciente y de la familia.

- Aplicar un plan terapéutico integral. Este plan incluye las actuaciones para cada síntoma y las orientadas al manejo de la situación terminal. Se deben fijar plazos para conseguir los objetivos, sin olvidar la prevención de síntomas nuevos que puedan aparecer.

- Tratamiento individualizado. Es importante comentar las opciones terapéuticas con el enfermo y aceptar su decisión, potenciando así su autonomía. La autonomía del paciente en la sociedad actual tiene cada vez mayor importancia y, por tanto, debe ser respetada y potenciada como un derecho del enfermo.

- Monitorización de los síntomas. Se recomienda el uso de instrumentos de medida estandarizados (escalas de puntuación y escalas analógico-visuales) y esquemas de registro adecuados (esquema corporal del dolor, tabla de síntomas...). Esto ayudará a clarificar objetivos, sistematizar el seguimiento y mejorar la calidad del trabajo mediante comparación de resultados.

- Atención a los detalles. La actitud del equipo, mediante la creación de un ambiente terapéutico cálido, alivia muchos síntomas físicos, aumentando su umbral de percepción del sufrimiento.

- Instrucciones correctas y completas. Explicar para qué sirve cada medicamento, los efectos secundarios que probablemente pueden aparecer y la manera adecuada de tomar cada uno de ellos contribuye a aumentar el cumplimiento terapéutico y la eficacia del tratamiento.

- Administración pautada. No es conveniente dar medicamentos a demanda, sino administrados a intervalos regulares según la duración de la acción de cada fármaco. Con esto se evita la reaparición del síntoma (dolor, vómitos) y se logra, por tanto, la mejora del bienestar del paciente.

- Revisión continúa del tratamiento. Deben tenerse en cuenta los efectos secundarios, las interacciones de fármacos y la sintomatología cambiante de los pacientes. Hay que utilizar siempre medicamentos de eficacia contrastada y que se conozcan bien. En pacientes de riesgo, como ancianos debilitados, enfermos con malnutrición y pacientes con fallos multiorgánicos, pueden aparecer efectos secundarios no previstos, entre los que se incluye la sobredosis.
- Promoción del confort. No hay por qué limitarse al uso de fármacos, ya que otras medidas terapéuticas como el frío, calor, masajes, relajación pueden mejorar también la calidad de vida del enfermo. La promoción del confort debe estar siempre presente en las decisiones, teniendo en cuenta que algunos esfuerzos "paliativos" bienintencionados, como la aspiración repetida de secreciones o la quimioterapia de dudosa eficacia, pueden provocar a veces más sufrimiento que confort. Por otro lado, medidas orientadas a la prolongación de la vida, como respiradores o fármacos vasoactivos, pueden ocasionar gran disconfort y deshumanizar la muerte.

La rigurosidad en el seguimiento de estos principios conseguirá mejorar el nivel de confort de los enfermos y, por tanto, mejorar su calidad de vida en su fase final (Gómez-Batiste et al., 2002).

\section{CP Y DECISIONES EN EL FINAL DE LA VIDA}

Durante toda nuestra vida, como personas, como integrantes de una sociedad, como profesionales, nos vemos obligados a tomar decisiones de muy diversa índole. Pero cuando llega el final cestamos preparados para tomar decisiones que afectan muy directamente a ese periodo de la vida? Cabe la posibilidad de que ese final se presente de forma brusca, inesperada (por una enfermedad aguda, por un accidente...), pero cuando la vida se extingue gradualmente como consecuencia de una enfermedad evolucionada, irreversible, sin respuesta a tratamiento (cáncer, enfermedad crónica de un órgano, demencia...), podríamos hacernos las siguientes preguntas: ¿querría prolongar mi vida conectado a una máquina cuando ya no sea consciente de estar en este mundo?, ¿querría que me alimentasen de forma artificial si ello implica prolongar la agonía?, ¿querría que me aliviasen el sufrimiento físico y psicológico, aunque ello llevara a acortar los últimos días?, ¿sería conveniente que tomase determinadas decisiones por adelantado?, ¿tengo derecho a tomar libremente las decisiones que van a afectarme más tarde mientras todavía soy competente?, ¿tengo derecho también a estar bien informado?

En determinados momentos puede resultar complicado dar respuestas a estas y a otras preguntas que nos pueden surgir. Son muchos los condicionantes culturales, educacionales, personales, religiosos y de otros tipos que nos van a influir y que deberían llevarnos a una profunda reflexión como personas y como profesionles; reflexión que debería, a su vez, conducirnos a buscar formación, información, ex- 
periencia y, en definitiva, a prepararnos para tomar las deciciones más razonables en este periodo final (Núñez Olarte, 2004).

\subsection{Una visión humanista}

La autobiografía de cada una de las personas es única e intransferible: está entretejida y condicionada por los acontecimientos vitales, por la posición social y económica, por las actitudes personales, por nuestro origen, por la genética, por los estilos de vida, por el lugar geográfico donde hemos nacido y vivido, por nuestras creencias y valores. Todo esto configura un modo de pensar, hacer y decidir que nos convierte en auténticos protagonistas no solamente de cómo vivimos, sino también de cómo deseamos morir. Existen, además, circunstancias especiales en las que la muerte puede ocupar parte de nuestros pensamientos: el fallecimiento de algún ser querido, una enfermedad grave, accidentes, catástrofes, etc. A través de todo ello se va configurando nuestro concepto individual sobre la vida y la muerte, que contribuye al pensamiento común sobre la muerte cuando tratamos de definir, aclarar y expresar cómo queremos que sea nuestro ir-muriéndonos. Es bien seguro que nos gustaría tener la ayuda competente de los profesionales de la salud para el control de los síntomas físicos y emocionales, una comunicación afectiva adecuada con nuestros seres queridos y una solidaridad natural y espontánea por parte de todos. Pero el requisito previo e indispensable para adoptar disposiciones e incluso dejar constancia escrita de las decisiones finales es tener asumida la muerte de una forma natural, como una parte del proceso de la vida a la cual hay que mirar de cara. Con esto, solo nos quedaría por resolver el "dolor de tener que morir". Decir adiós y desaparecer nos va a causar tristeza e inquietud. Llegado el momento, seguro que a todos nos quedan muchas cosas por hacer. Por tanto, ante lo inevitable, será mejor poner nuestro empeño en vivir bellamente para que podamos poder morir de una manera hermosa, serena y sin miedo: aceptando la propia muerte (Sanz Ortiz, 2001).

Una parte importante en este proceso de aceptación tiene que ver con la búsqueda de la autorrealización y con la dimensión espiritual propia del ser humano. Y una característica esencial de la espiritualidad es su componente experiencial y subjetivo (Fombuena Moreno, 2014). A la espiritualidad se llega desde la vivencia $y$, por tanto, la experiencia espiritual se enriquece con múltiples matices individuales que se explican desde la historia personal de cada sujeto. Tomar conciencia de esta historia personal, contemplarla y aceptarla, facilitará el reconocimiento del ser espiritual y de su desarrollo, alimentándolo de nuevas experiencias y formas distintas a las vividas con anterioridad. La atención adecuada a la espiritualidad de la persona en el final de su vida precisa de cierta sensibilidad para detectar las necesidades del paciente en este terreno, lo cual presupone, como ejes principales de la relación entre el personal sanitario y el paciente, disposición a la escucha y respeto a las creencias y valores.

\subsection{Una vision desde la lex artis}

Reconocer si el enfermo se encuentra en la fase final de su vida no es una cuestión baladí. La medicina no es una ciencia exacta, nos movemos entre ciertos márgenes de incertidumbre; para disminuir esta, los profesionales sanitarios deben basar sus actuaciones y decisiones en la evidencia disponible, estableciendo criterios que nos ayuden a determinar el final de la vida a consecuencia del avance inexorable de la enfermedad (Sanz Rubiales et al., 2006).

Por enfermedad en fase avanzada entendemos aquellas entidades nosológicas con estadios clínicos de la enfermedad establecidos y no reversibles tras un periodo de tratamiento estandarizado. Esta situación de enfermedad avanzada llegará a la fase terminal. Es decir, estamos ante una enfermedad avanzada, progresiva e incurable, con falta de posibilidades razonables de respuesta al tratamiento específico, con numerosos problemas o síntomas intensos, múltiples, multifactoriales y cambiantes, gran impacto emocional en el paciente, familia y equipo, con pronóstico de vida limitado.

Las posibilidades de diagnóstico y terapéuticas que el desarrollo científico-tecnológico pone a nuestra disposición pueden salvar vidas, pero también, en determinadas circunstancias, pueden llevar a una obstinación continuada que impida morir en paz. En consecuencia, forma parte de la lex artis el limitar las actuaciones diagnóstico-terapéuticas, lo cual obliga a reconocer cuándo el enfermo se halla en el final de la vida como consecuencia de su enfermedad, a predecir las consecuencias que se derivan de la continuación o no de las actitudes terapéuticas y a conocer los deseos del paciente. En esta línea nos ayudará a establecer unos criterios de actuación el determinar si una medida diagnóstico-terapéutica es o no adecuada teniendo en cuenta la situación clínica del paciente y las consecuencias que se prevén ante su utilización. Para ello nos apoyaremos en los principios de: proporcionalidad (evaluar los efectos positivos y los negativos), futilidad (utilidad de los tratamientos, beneficio esperado $<5 \%$ ) y calidad de vida (sentida y esperada por el paciente). 
Algunos síntomas, como el dolor, la disnea y otros, constituyen una experiencia desagradable que lleva a buscar su alivio con todas las armas médicas a nuestro alcance, aunque ello suponga aceptar efectos secundarios no deseados ni buscados. Es lo que se conoce como el "principio del doble efecto", que está considerado como buena praxis médica y ética. Incluso la sedación en el final de la vida puede estar perfectamente indicada como actitud terapéutica para el alivio de síntomas y de situaciones refractarias a otros tratamientos debidamente administrados. En esta tesitura, surgen inevitablemente preguntas cuya respuesta nos indicará si la medida es correcta desde el punto de vista de la lex artis y de la ética: ¿se han utilizado todos los tratamientos disponibles alternativos a la sedación?, ¿de quién es el sufrimiento que se pretende aliviar, del enfermo, de la familia o del equipo profesional?, ¿quién toma la decisión?, ¿al sedar de manera irreversible a un paciente se la ha arrebatado su condición de persona? Los complejos problemas que se derivan de la puesta en práctica de la sedación al final de la vida obligan al profesional a utilizar protocolos que aseguren la buena práctica clínica y el respecto a los derechos y a la dignidad de la persona (Gómez-Batiste, Borell Busquets y Roca Casals, 2003).

Pero, en todo caso, hemos de recordar que la sedación paliativa al final de la vida busca la paliación de los síntomas refractarios que están generando un enorme sufrimiento a la persona; no pretende directamente el acortamiento de la vida, aunque este pueda producirse en algunos casos como efecto indirecto no buscado, por ello es una práctica legítima desde el punto de vista ético y de la lex artis.

Otro problema de complicado manejo es el que se refiere a la alimentación e hidratación procuradas de forma no natural cuando el enfermo pierde la facultad de deglutir y se prevé un pronóstico vital incierto. Esta práctica sigue generando debate y preguntas éticas a la comunidad científica y a la sociedad: ¿se han tenido en cuenta los deseos del paciente?, ¿son la alimentación e hidratación cuidados básicos que no pueden ser omitidos? O bien ¿son procedimientos que forman parte de los tratamientos médicos y sujetos, por tanto, a una indicación con unos beneficios esperados que no se llegan a obtener? De la respuesta a estas cuestiones dependerá que consideremos las medidas de alimentación e hidratación, o su retirada, como acciones concordantes o no con la lex artis. En general, si el enfermo está en situación de agonía irreversible, en estado de semi o total inconsciencia, no estaría indicada una alimentación artificial, liquida, por sonda nasogástrica, ya que no le aportaría ningún tipo de beneficio y sí podría causarle mayor disconfort ya que en ese estado el organismo no asimila.

La situación es diferente cuando el paciente tiene un pronóstico de vida previsible de semanas o meses, y se encuentra en mejor estado; en esa situación hay que valorar los pros y los contras antes de tomar una decisión al respecto y siempre teniendo en cuenta los deseos del paciente.

Por último, la adecuación a la lex artis de otras medidas terapéuticas, como pueden ser la administración de antibióticos, las transfusiones o la ventilación mecánica no invasiva (VMNI), dependerá del juicio de proporcionalidad sobre las mismas, establecido tras valorar los beneficios que se desean obtener y los posibles riesgos en un enfermo particular y concreto (Sanz Ortiz, 1997).

\subsection{Una visión ética}

En las últimas décadas del siglo XX la ciencia médica ha experimentado un avance espectacular en el campo de la terapéutica, la revolución tecnológica y el descubrimiento del código genético. Esta evolución de la medicina ha cambiado, en gran medida, la vida de los seres humanos. Las medidas preventivas son más efectivas y la batalla contra la enfermedad más eficaz; todo ello ha contribuido a alargar la expectativa de vida, a pesar de lo cual siempre llegará el momento en que nos enfrentemos al proceso de morir. Es preciso, por tanto, reflexionar sobre las demandas que plantea una sociedad llena de nuevos retos tecnológicos, humanos y éticos, integrada por personas con diferentes visiones de su proyecto vital y distinta filosofía sobre la vida y la muerte. Por estas razones, la asistencia sanitaria, cuyos protagonistas son los pacientes y sus familias, los profesionales y la sociedad en general, plantea en la actualidad cada vez más problemas que no solo están relacionados con la práctica médica, sino también con el mundo jurídico y el complejo mundo de los valores éticos.

La práctica de la medicina está constituida por una continua toma de decisiones de orden científico-técnico y de orden moral o ético, siendo su objetivo prevenir y curar las enfermedades, cuidar de la salud y aliviar el sufrimiento. El progreso biológico, farmacológico y tecnológico aplicado a la práctica clínica diaria ha planteado nuevos problemas asistenciales antes inexistentes. Ha sido por ello necesario desarrollar un nuevo marco en la relación del profesional sanitario con el paciente, salvaguardando siempre los intereses del enfermo, y respetando también los conocimientos técnicos y los valores del profesional. 
Pues bien, la disciplina que analiza la conducta humana, examinada a la luz de los valores y principios morales, en el campo de las ciencias de la vida y del cuidado de la salud, es la ética médica o bioética. La ética nos ayuda a encontrar nuestro horizonte de plenitud desde la reflexión, mejorando nuestras posibilidades de realización y nuestro saber práctico. $Y$ en particular, la ética aplicada a un campo concreto de la actividad humana -en nuestro caso, el sanitario- tiene que ser capaz de aplicar las normas y valores a ese ámbito de la vida y de la profesión. Los valores son aquí tan importantes como los hechos. El establecimiento científico de los hechos, como condición previa, da corrección a las decisiones médicas, mientras que los valores les dan calidad. Pero cabe recordar que, con ser importantes las normas y los valores en abstracto, a tomar decisiones prácticas no se aprende solo con el saber general, ya que en cada uno de los pacientes hay que integrar dicho saber en lo concreto y particular. Se trata de tener capacidad de juicio para tomar decisiones con los pacientes sobre el final de su vida.

Es sabido que en 1979 Tom L. Beauchamp y James Childress formularon la teoría de los cuatro principios, que sigue siendo hoy día el paradigma bioético dominante en el mundo sanitario. Como modelo principalista, se centra en la acción o praxis, y otorga gran relevancia al proceso de toma de decisiones efectuado mediante la racionalidad argumentativa. El profesional sanitario tiene la obligación de no hacer daño, según el principio ético de no maleficiencia, que delimita su campo de actuación. Este principio hace referencia a la vida biológica que el personal sanitario tiene entre sus manos y al respeto que esta merece. También está obligado a procurar los medios diagnósticos y terapéuticos mas adecuados a cada situación para conseguir el mayor beneficio del paciente, según el principio de beneficencia. El otro sujeto de esta relación, el enfermo, aporta un nuevo principio, el de autonomía. Por autonomía se entiende la capacidad de realizar actos con conocimiento de causa, con información suficiente y en ausencia de coacción interna o externa. Desde su autonomía, la persona enferma debe intervenir en el proceso para ir decidiendo, conjuntamente con el profesional, qué es beneficioso para él. Esta es la razón por la que ambos principios, el de autonomía y el de beneficencia, resultan inseparables: no es posible hacer el bien a los otros en contra de su voluntad.

En toda relación sanitaria existe un sujeto que, si bien no está presente físicamente, interviene de una forma u otra: la sociedad. Los recursos humanos, profesionales y técnicos son generados por la sociedad, y los profesionales tienen la obligación de distribuirlos equitativamente y de evitar cualquier tipo de discriminación en el acceso a los mismos. Todo ello viene exigido por el principio de justicia.

Este marco normativo parece claro, hasta que nos enfrentamos con la realidad clínica. Los principios éticos son criterios o normas de conducta que introducen la garantía de una racionalidad argumentativa en la toma de decisiones pero por sí solos no son suficientes. Su aplicación a la realidad no es automática, sino que exigen un proceso de deliberación posterior que permita discernir cuál es la mejor manera de respetarlos en cada uno de los posibles casos clínicos (Cruceiro, 2004).

En un paciente en el final de su vida, que recibe cuidados paliativos, son múltiples los factores que hay que tener en cuenta en la toma de decisiones, por ejemplo, aquellos relacionados con la evolución de la enfermedad (estado general, complicaciones, pronóstico...), o los que atañen a la proporcionalidad de la intervención clínica (cálculo ponderado de riesgos y beneficio esperado) o aquellos relativos a los fines y objetivos que se persiguen. Además, hemos de tener en cuenta que afloran sentimientos y actitudes emocionales ante los procesos de muerte de los pacientes que no deben influir en nuestras decisiones. Es muy importante que los juicios morales no se reduzcan a un mero sentimiento emocional, por ello es necesaria una reflexión racional que oriente ese sentimiento a una deliberación razonada.

En el proceso de toma de decisiones al final de la vida los profesionales sanitarios serán más efectivos y acertados en sus respuestas a retos éticos si aprenden a abordarlos de forma sistemática para llegar a decisiones prudentes o razonables. El primer paso es el análisis de los hechos, lo cual nos obliga a una revisión detallada de la historia clínica del paciente, a evaluar la situación actual y los objetivos que hay que plantearse para así establecer, en base a ellos, unos posibles cursos de acción, como pueden ser la indicación o no de determinadas medidas terapéuticas (imprescindible revisar la evidencia disponible sobre el tema) y siempre tras conocer los puntos de vista del paciente y de sus familiares. Si en las decisiones que se deben tomar surge conflicto de valores, habrá que identificarlos y analizarlos para establecer posibles cursos de acción.

\subsection{Una visión legal y jurídica}

Solo al paciente le corresponde tomar decisiones que afectan a su propia vida. La participación y la decisión del enfermo son una ayuda inestimable en las situaciones de incertidumbre. Hay que determinar, a tal efecto, la 
competencia o capacidad del paciente, es decir, hemos de averiguar si este puede tomar una decisión o no, si comprende la información recibida y si puede tomar decisiones sobre la base de esa información suministrada. Cuando el enfermo no es competente, se deberá identificar a un sustituto en la toma de decisiones. En la práctica suele ser un miembro de la familia. El representante $o$ tutor sustituye al enfermo en la toma de decisiones pero siempre basándose en el mejor conocimiento de los valores del enfermo y de lo que se ajuste mejor a los intereses de este. Estas decisiones se denominan decisiones de sustitución o decisiones subrogadas.

En cualquier caso, ya sea directamente o a través de representante o tutor, la voluntad del paciente y sus intereses han de hacerse presentes a través del consentimiento informado. El consentimiento informado es un proceso de información y de comunicación entre el profesional sanitario y el enfermo, que puede culminar con la autorización del paciente (o de su representante) a las actuaciones diagnóstico-terapéuticas de las cuales el médico ha ponderado riesgos, beneficios y alternativas. Para la bioética principialista el consentimiento informado se fundamenta en dos principios: la obligación del profesional de respetar las decisiones autónomas de los pacientes, así como de procurar el mayor bien a dichos pacientes y de ayudarles a realizar en lo posible su propio proyecto vital. Se establece, pues, la obligación de realizar un proceso adecuado de consentimiento informado, solidamente fundamentado, tanto ética como jurídica y deontológicamente.

Es necesario, pues, que los profesionales dediquen tiempo suficiente a la información y comunicación, lo cual les dará la oportunidad de conocer los valores del paciente, así como su concepto de calidad de vida. En el proceso final de la vida la información debe responder a las necesidades del paciente, a la que él mismo solicite o sea capaz de soportar. Para llegar a conocer dichas necesidades de información es imprescindible saber escuchar. Hay que identificar, así, las diferentes necesidades de información propias de cada enfermo. Conviene entonces valorar la conveniencia o no de la abstención justificada (privilegio terapéutico). Y a partir de ahí, es preciso proporcionar la información teniendo en cuenta ya las necesidades y características del paciente, su biografía y las aportaciones que pueda hacer la familia. El objetivo de todo el proceso informativo es el de potenciar la autonomía del enfermo (véase Sociedad Española de Cuidados Paliativos SECPAL, 2016).

En este sentido, la ley general de sanidad en su artículo 10 destaca de manera clara la importancia del respeto a la autonomía de las personas enfermas. Tal respeto ha esta- do tradicionalmente muy lejos de los valores de nuestro entorno sociocultural y profesional, en el que ha destacado el paternalismo en la relación profesional sanitario-paciente. Pero ha pasado el tiempo suficiente para permitir el desarrollo de nuevas leyes, tanto en el ámbito estatal como autonómico, que reafirman el hecho de que el titular del derecho a la información es el paciente.

El médico es el responsable de garantizar ese derecho. Las personas vinculadas al paciente, por razones familiares o de hecho, solo serán informadas en la medida en que el paciente lo permita de manera expresa o tácita. Las leyes referidas contemplan dos instrumentos principales como garantes de la autonomía: el proceso de consentimiento informado, en el caso del paciente capaz, y el documento de voluntades anticipadas, cuando el paciente se encuentre en situación de incapacidad.

Los profesionales sanitarios y las organizaciones a las que pertenecen deben velar activamente para que este derecho fundamental de los pacientes sea respetado, procurando así una atención con un nivel adecuado de excelencia moral. Para que se cumplan las exigencias éticas y jurídicas del consentimiento informado han de respetarse unos elementos normativos:

- Que sea un proceso continuo, dialógico (hablado), comunicativo, deliberativo y prudencial. En ocasiones requiere apoyo escrito y siempre debe ser registrado de forma adecuada. No se trata, pues, de un hecho aislado centrado en un formulario que el enfermo tiene que firmar.

- Voluntariedad, es decir, se trata de un acto libre, no coaccionado ni manipulado.

- Información suficiente, que incluya no solo la naturaleza, objetivos y posibles efectos secundarios de la acción o decisión que hay que llevar a cabo, sino también las diferentes opciones alternativas existentes, con sus respectivos beneficios y riesgos.

- Información comprensible, adaptada a las peculiaridades socioculturales del paciente, haciendo uso correcto del lenguaje verbal, no verbal y escrito.

- Capacidad por parte del paciente para comprender la información, evaluarla y comunicar su decisión.

Finalmente, tras el proceso de deliberación, el paciente toma una decisión que es de aceptación o de rechazo de las medidas diagnóstico-terapéuticas propuestas por el profesional. Dicha decisión marca, con frecuencia, el punto de partida de un nuevo proceso de deliberación y toma de decisiones. Las herramientas de aplicación práctica que permiten realizar un proceso correcto de consentimiento informado son: 
- Técnicas de entrevista clínica, soporte emocional y relación de ayuda.

- Protocolos de evaluación de la capacidad de la persona, que nos permitan establecer cuándo un paciente está en condiciones de tomar decisiones.

- Formularios escritos de consentimiento informado que sirvan para apoyar el proceso oral, para ser registrados y, en su caso, para probar legalmente que se dio información y se obtuvo el consentimiento.

En cuidados paliativos la finalidad y los objetivos del consentimiento informado permanecen inalterados pero la metodología, estructura y proceso pueden presentar ciertas dificultades derivadas de la especial vulnerabilidad de la persona en fase avanzada de su enfermedad con una expectativa de vida corta. Son muchos los pacientes que, llegados a esta fase al final de la enfermedad, atendidos por equipos de cuidados paliatios, presentan un grado tal de deterioro físico, cogntivo o emocional que se hace muy difícil establecer una adecuada comunicación para dar información y plantear las decisiones que hay que tomar. Cuando esto ocurre hay que recurrir a la familia para informar y averiguar los valores, deseos y condicionamientos expresados por el paciente durante la trayectoria de su vida y enfermedad, a fin de tomar las decisiones en base a ellas.

Consideremos ahora la cuestión de las voluntades anticipadas. El documento de voluntades anticipadas (Broggi, 2001) permite a una persona mayor de edad y con capacidad suficiente, de manera libre, participar en sus cuidados y planificar su futuro mediante la expresión de sus deseos, para cuando no sea capaz de tomar decisiones por sí mismo y otros deban hacerlo en su lugar. Puede incluso designar en él a un representante como interlocutor válido. Dicho documento nos permitirá conocer el concepto de calidad de vida del paciente, y contribuirá a disminuir la tensión que supone el tomar decisiones en condiciones de incertidumbre.

A falta de un documento de voluntades anticipadas, la tensión se produce especialmente cuando el enfermo no está capacitado para tomar decisiones y no existe un acuerdo entre sus representantes, o entre estos y los profesionales sanitarios, a la hora de tomar una decision.
Todas las personas que participan en la relación clínica deberían esforzarse entonces por conocer qué hubiera deseado el enfermo en esas circunstancias concretas. Pero a menudo no resulta fácil, dada la complejidad de las decisiones que hay que tomar. Sustituir las decisiones de otra persona, sobre todo en el final de su vida, entraña una enorme responsabilidad. Si las opiniones discrepan abiertamente y no se puede alcanzar un consenso, existe la opción de consultar al Comité de Ética Asistencial (CEA). Se trata de una comisión consultiva e interdisciplinar al servicio de los profesionales y usuarios de una institución sanitaria, creada para analizar los posibles conflictos éticos que se producen a consecuencia de la labor asistencial y asesorar en su resolución. El objetivo principal del CEA es el de mejorar la calidad ética de la asistencia sanitaria. EI CEA trata de facilitar el proceso de decisión en los casos más conflictivos, asesorando a los profesionales para que puedan tomar la decisión más prudente posible teniendo en cuenta todos los valores implicados.

\section{CONCLUSIÓN}

La ética nos ayuda a encontrar nuestro horizonte de plenitud desde la reflexión, mejorando nuestras posibilidades de realización y nuestro saber práctico. La ética enfocada hacia un campo concreto de la actividad humana, el de la atención en el final de la vida, tiene que ser capaz de aplicar las normas y valores a ese ámbito de la vida y de la profesión. Vemos, así, que la responsabilidad por el ser humano y el vínculo que se establece con la persona que muere crea obligaciones para con esa persona: cuidarla es tanto como reconocer en ella su dignidad y diferencia. Pero a tomar decisiones no se aprende solo con el saber general, ya que a cada uno de los pacientes hay que integrarlo en lo concreto y particular. Se trata de desarrollar, también desde la experiencia de la práctica diaria, la capacidad de juicio para tomar decisiones con los pacientes sobre el final de su vida. En este sentido, y como conclusión, podríamos afirmar que los CP representan la respuesta de las ciencias de la salud al imperativo ético de ayudar al enfermo y a su familia a afrontar la experiencia del sufrimiento antes la muerte desde una perspectiva holística donde se integran la dimensión física, psíquica, social y espiritual del ser humano. Conseguir que el paciente, a pesar de su enorme fragilidad, pueda vivir plenamente los últimos momentos de su vida en función de sus valores, es el gran reto de la medicina paliativa de este siglo. 


\section{BIBLIOGRAFÍA}

Broggi M. A. (2001). El documento de voluntades anticipadas. Medicina Clínica, 117 (1), pp. 14-15. https://doi. org/10.1016/S0025-7753(01)71996-3

Cassel, E. J. (2004). The nature of suffering and the goals of medicine (2. $\underline{\text { ed.). }}$ New York: Oxford University Press. https://doi.org/10.1093/acprof:o so/9780195156164.001.0001

Centeno Cortés C., Gómez Sancho M., Nabal Vicuña M. y Pascual López A. (eds.) (2009). Manual de medicina paliativa. Pamplona: EUNSA.

Connors, A. F., Dawson, N. V., Desbiens, N. A., Fulkerson, W. J., Goldman, L. Knaus, W. A. [...] y Hakim, R. (1995). A controlled trial to improve care for seriously ill hospitalized patients: The Study to Understand Prognoses and Preferences for Outcomes and Risks of Treatments. JAMA, 274 (20), pp. 1591-1598. https://doi.org/10.1001/ jama.1995.03530200027032

Cruceiro A. (2004). Ética en cuidados paliativos. Madrid: Triacastela.
Fombuena Moreno, M. (2014). Situación clínica de enfermedad avanzada y espiritualidad. [Tesis doctoral inédita]. Universidad de Valencia: Valencia.

Gómez-Batiste X., Borrell Busquets R. Y Roca Casals, J. (2003). Ética clínica y cuidados paliativos. JANO, LXV (1495), pp. 52-55.

Gómez Batiste, X., Mata, I. de la, Fernández, M., Ferrer, J. M., García, E., Novellas, A. [...] y Sunol, R. (2002). Guía de Criterios de Calidad en Cuidados Paliativos. Sociedad Esapañola de Cuidados Paliativos. Ministerio de Sanidad y Consumo.

Núñez Olarte J. M. (2004). Una elección básica: tratamiento paliativo o tratamiento curativo. En: Cruceiro, A. Ética en cuidados paliativos. Madrid: Triacastela, pp. 109-125.

Sanz Ortiz, J. (1997). Decisiones en el final de la vida. Medicina Clínica, 109 (12), pp. 457-468.

Sanz Ortiz, J. (2001). Final de la vida: ¿puede ser confortable? Medicina Clíni- ca, 116 (5), pp. 186-190. https://doi. org/10.1016/S0025-7753(01)71765-4

Sanz Rubiales A., Valle Rivero, M. L. D., Flores Pérez, L. A., Hernansanz de la Calle, S., Gutiérrez Alonso, C., Gómez Heras, L. y García Recio, C. (2006). Actitudes ante el final de la vida en los profesionales de la sanidad. Cuadernos de Bioética, XVII (2), pp. 215-236.

Sociedad Española de Cuidados Paliativos SECPAL (2016). Directorio de Recursos de Cuidados Paliativos en España. Directorio SECPAL 2015. Monografías SECPAL, n.o 8. [En línea]. Disponible en http://www.secpal. com//Documentos/Blog/monografia8_directorio.pdf

\section{Otros recursos}

Organización Mundial de la Salud. Control del cáncer: Cuidados paliativos. Guía de la OMS para desarrollar programas eficaces. [En línea]. Disponible en https:// www.who.int/cancer/publications/cancer_control_palliative/es/ 